\title{
Interactions Between Physiological and Affective Arousal: A Laboratory Exercise for Psychology
}

\author{
David L. Rowland \\ Anneli Kaariainen \\ Elisabeth J. Houtsmuller \\ Valparaiso University
}

\begin{abstract}
As part of the laboratory component of an introductory psychology course, 420 students participated in an exercise demonstrating the relation between psychological states and physiological response. Before and during 2 videotape segments, the first relaxing and the second suspenseful, student experimenters measured the heart rate and blood pressure of fellow students who served as participants. Participants also completed 4 psychoaffective items aimed at assessing their subjective arousal to the video stimuli. Both physiological and subjective arousal measures showed significant variation across stimulus conditions, demonstrating the psychophysiological nature of affective arousal. Student evaluation of the laboratory exercise was positive with respect to learning goals, interest in the project, and clarity of procedures.
\end{abstract}

Conveying the importance of the relationship between biology and behavior and mental events is generally included among the goals of an introductory psychology course. Yet, this relationship is often detailed and complex, making its comprehension difficult for the beginning student. Seldom can students draw on their personal experiences to augment their understanding of biopsychological relations. Even within the framework of a psychology lecture or laboratory course, practical demonstration of such relationships is often restricted to computer simulations or ones acted out by groups of students (Hamilton \& Knox, 1985; Solomon, Cooper, \& Pomerleau, 1988) or to making inferences about unobservable physiological mechanisms from overt behavior (Harcum, 1988). Nonsimulated exercises may require sophisticated equipment (e.g., polygraph), thereby limiting participation to only a few students (Webster \& Muir, 1995), or they may require laboratory animals (Kemble, 1983; Wellman, 1984, 1985), an option not available at many institutions. Accordingly, students of introductory psychology may receive little or no exposure to experiential aspects of biopsychological interactions. Thus, they are deprived of the insight afforded by such hands-on learning experiences and may be less appreciative of the relevance of such interactions to their daily functioning.

We describe a psychophysiological exercise that demonstrates the relation between biological and psychological phenomena in humans yet requires little expertise on the part of the instructor. Psychophysiology, a subfield of biopsychology, attempts to relate subjective mental states (e.g., anxiety) to variation in physiological parameters such as heart rate (HR) and blood pressure (BP; Greenwald, Cook, \& Lang, 1989). Unlike most biopsychological relationships studied in psychology that emphasize the influence of biology over behavior, psychophysiological analysis presents a unique opportunity to explore the influence of psychology (e.g., mental states) on physiology. Moreover, with recent advances in biotechnology, such phenomena can be readily demonstrated in fairly large groups of human participants at modest cost.

In the exercise described here, we presented students with audiovisual stimuli intended to induce varying levels of psychological arousal and, hence, autonomic activation. The purpose was to demonstrate the integral connection between psychological interpretation and reaction to stimuli and concomitant physiological response.

\section{Method}

\section{Participants}

Four hundred twenty students, enrolled in the optional laboratory component of an introductory psychology course for natural science credit, participated in this exercise. These students had enrolled in 5 or 6 separate lab sections over each of three consecutive semesters of the course $(n=$ 114,128 , and 178 , respectively, each semester; 25 to 30 in each lab section).

\section{Stimuli}

We used two 10-min videotape segments as stimuli, one intended to induce relaxation, the other suspense and excitement (see the Notes section for details). The relaxation segment, taken from Meditation (1990), consisted mainly of sights and sounds of moving water. To determine the appropriate stimulus for suspense, two lab assistants viewed 10 "suspense" films and prepared segments from 4 that were then pretested on 15 students (not enrolled in this course). Students rated these segments on six standard affective and arousal dimensions. The video segment having the highest overall rating for the six items combined (i.e., Fatal Attraction, Jaffe, Lansing, \& Lyne, 1987) was selected for the lab exercise. 
Response Measures

Before and during each stimulus segment, student experimenters measured their fellow students' HR and BP (both diastolic and systolic), autonomic parameters influenced by emotions and arousal (Lang, Levin, Miller, \& Kozak, 1983), using portable BP monitors (Omrom, Model HEM-402c, Vernon Hills, IL, approximately $\$ 30$ each). At the end of each video segment, participants responded to four items assessing their level of excitement and arousal on 7-point scales ranging from 1 (not at all) to 7 (extremely): relaxed, excited, calm, and fearful.

\section{Procedure}

The exercise, sequenced to coincide with lecture material on motivation and emotion, required approximately 1 hr within a 2-hr lab period. One week before the lab, the instructor asked students to refrain from eating, drinking, or smoking $15 \mathrm{~min}$ prior to the next scheduled lab session (because data collection did not commence until $45 \mathrm{~min}$ into the lab period, a 1-hr abstinence period was in effect). The lab began with a demonstration of how to use the BP monitors. Students were then divided into experimenter-participant pairs, with participants equally divided between men and women (sex of the experimenters was not controlled). To gain practice, the experimenters took three consecutive $\mathrm{BP}$ and HR readings from the nondominant arm of participants.

Because the BP monitors required a minimum 12-min interval between readings to ensure accuracy, the lab instructor used the next $30 \mathrm{~min}$ for other lab activities before actual data collection began. This interval included a short presentation (about $15 \mathrm{~min}$ ) regarding relationships between biological and behavioral and cognitive systems without making specific reference to the nature or content of the ensuing exercise. The instructor then informed the students about the physiological and psychoaffective measures they would be taking and that they would take these measures before and during or after watching several videotapes.

At this point, the instructor semidarkened the room and asked the experimenters to collect and record baseline physiological measures from the participants while they completed, in confidence, their ratings on the subjective arousal items. After $3 \mathrm{~min}$, the first of the two stimulus segments was presented on a 25-in. monitor. To maximize stimulus effects, the relaxation segment preceded the suspense segment. Toward the end of each segment (at $9.5 \mathrm{~min}$ ), the instructor signaled the experimenters to again collect physiological measures, and immediately after the segment ended, the participants rated, again in confidence, their subjective reaction to the stimulus. About 6 min separated stimulus segments, during which students remained seated but were permitted to converse. The instructor collected and analyzed the data and prepared summarized results for discussion in the subsequent lab period.

Two weeks later, all students, both experimenters and participants, evaluated the lab exercise on items addressing learning goals and procedural aspects of the exercise. In addi- tion, two open-ended questions queried students about what they liked most and least about the exercise.

\section{Results}

\section{Effect of Stimulation}

Because students formed experimenter-participant pairs for this exercise, data were obtained from only half of them $(n$ $=210$ ). Selected mean physiological responses and subjective ratings during baseline and to the stimulus segments appear in Table 1 . A $3 \times 2$ ANOVA using stimulus condition (baseline, relaxation, suspense) and sex of the participant as factors yielded the following effects. For stimulus condition, all physiological and subjective arousal measures showed significant variation, $F(2,205) \geq 74.6, p \leq .001$. These measures indicated lowest arousal during relaxation, highest during suspense, and intermediate at baseline. Post hoc analyses using protected $t$ tests indicated that the relaxation and suspense conditions differed from each other for all variables $(p$ $<.05)$, although these two conditions did not always differ from baseline. Sex effects occurred on two physiological measures, with women showing lower diastolic and systolic BP than men, $F(1,205) \geq 6.91, p \cong .009$. On subjective measures, women reported higher levels of arousal than men; specifically, they indicated less relaxation and calmness and greater fear and excitement, $F(1,205) \geq 4.43, p \leq .037$.

\section{Evaluation of the Lab}

Results of the student evaluation of the lab exercise appear in Table 2. On all items, average ratings were nearer the positive end of the scale. For the two open-ended questions, most responses fell into one of four broad categories. Aspects that

\section{Table 1. Physiological and Subjective Measures Under Different Types of Stimulation}

\begin{tabular}{lccc}
\hline Measure & Baseline & Relaxation & Suspense \\
\hline $\begin{array}{l}\text { Physiological } \\
\text { Heart rate }\end{array}$ & & & \\
$\quad$ Men & 72.8 & 66.7 & 75.7 \\
$\quad$ Women & 72.8 & 67.6 & 79.3 \\
Blood pressure & & & \\
$\quad$ Men & 113.3 & 106.4 & 122.1 \\
$\quad$ Women & 103.1 & 96.1 & 114.4 \\
Subjective & & & \\
Relaxed & & & \\
$\quad$ Men & 5.5 & 6.3 & 3.9 \\
$\quad$ Women & 5.1 & 6.1 & 2.8 \\
Excited & & & \\
$\quad$ Men & 1.9 & 1.5 & 4.2 \\
$\quad$ Women & 2.2 & 1.4 & 4.8 \\
Fearful & & & \\
$\quad$ Men & 1.3 & 1.2 & 3.1 \\
$\quad$ Women & 1.5 & 1.2 & 4.5 \\
\hline
\end{tabular}

Note. Subjective arousal items were rated on a 7-point scale ranging from 1 (not at all) to 7 (extremely).

${ }^{a}$ Measured in beats per minute. ${ }^{b}$ Systolic $\mathrm{mm} \mathrm{Hg}$ (mercury). 
Table 2. Student Evaluation of the Exercise

\begin{tabular}{|c|c|c|c|c|c|c|}
\hline \multirow[b]{2}{*}{ Item } & \multicolumn{2}{|c|}{ Participant } & \multicolumn{2}{|c|}{ Experimenter } & \multicolumn{2}{|c|}{ Overall } \\
\hline & M & $S D$ & $M$ & $S D$ & $M$ & $S D$ \\
\hline Beneficial learning experience & 4.9 & 1.1 & 5.1 & 1.1 & 5.0 & 1.2 \\
\hline Increase understanding of biopsychology & 4.9 & 1.2 & 4.9 & 1.3 & 4.9 & 1.2 \\
\hline Increase knowledge of biopsych interactions & 5.0 & 1.1 & 5.0 & 1.3 & 5.0 & 1.2 \\
\hline Stimulate interest in more study & 4.7 & 1.4 & 4.6 & 1.4 & 4.6 & 1.4 \\
\hline Recommend using in future labs for this course & 5.3 & 1.2 & 5.2 & 1.2 & 5.3 & 1.3 \\
\hline Instructions clear and helpful & 5.8 & 1.2 & 5.7 & 1.4 & 5.8 & 1.3 \\
\hline Equipment difficult to use & 2.0 & 1.6 & 2.0 & 1.7 & 2.0 & 1.6 \\
\hline
\end{tabular}

Note. Items were based on a 7-point scale ranging from 1 (not at all) to 7 (extremely).

the students liked most about the exercise included (a) the realization that psychological factors and emotions influence physiology (49.3\%), (b) the interesting nature of the topic with the subsequent desire to learn more $(22.6 \%)$, (c) the enjoyment of working with and taking BP (15.9\%), and (d) the relevance of the exercise to real life (12.1\%). Aspects liked least included (a) the seemingly long and boring relaxation tape $(47.9 \%)$, (b) the length of the experiment $(21.4 \%)$, (c) the scariness of the suspense tape (15.0\%), and (d) problems that occurred in working with the BP equipment (9.5\%).

\section{Discussion}

The experimental parameters defined previously were sufficient to reveal significant changes in both physiological and subjective measures of arousal and thus provided a practical demonstration of the interaction between biological and psychological systems in humans. Although we implemented this particular module in our introductory course, adaptation to other lab or lecture courses that cover topics on relationships involving physiology, arousal, and emotions (e.g., motivation and emotion, physiological psychology) is certainly feasible. Student evaluations suggested that the exercise achieved the intended goals: Students described the exercise as a beneficial learning experience that increased their understanding of biological-psychological interactions. Furthermore, they seemed to enjoy the opportunity to investigate such relationships within themselves. Given that over $90 \%$ of the students were majoring in the humanities, business, nursing, and other fields of science, we were encouraged by the sizable portion of the students expressing the desire to learn more about the topic.

In addition to the pedagogical goals achieved through students' participation in the exercise, the data have potential to stimulate interest and discussion among students. For example, beyond the anticipated stimulus effects, we found that women in our sample consistently verbalized stronger subjective emotional and arousal responses than men, although they did not show stronger physiological responses. Such findings provide ample opportunity to explore the data in greater depth and to challenge students to generate explanatory hypotheses. For instance, men may be less aware of their emotional states than women or perhaps less willing to admit to them (e.g., Kirkpatrick, 1984; Pierce \& Kirkpatrick, 1992). Alternatively, ceiling effects may cap physiological responding long before subjective arousal peaks, thereby ob- scuring sex differences during suspense (Rowland, 1995). Finally, although the concept of statistical interaction exceeded the scope of our introductory-level course, students could often intuitively appreciate, raise questions about, and generate explanations for various sex-stimulus interactions based on their own gender stereotypes (or resistance to them) about emotional responding.

As with any lab exercise, several practical issues require comment. We summarized data over 5 or 6 lab sections each semester ( $n=60$ to 80 ) to increase statistical power. However, even when analyses of individual sections lacked statistical significance because of small samples (e.g., during summer session), expected trends for stimulus effects have been borne out. One way to augment sample size is to have all students serve as participants (collecting data on themselves), assuming an adequate supply of BP monitors.

A second concern relates to the cost and use of the BP monitors. At $\$ 25$ each (bulk discount price), we spent $\$ 400$ for the monitors. Although seemingly high, such costs are not unusual within the context of an introductory lab course. Site licenses for software exercises in perception and memory typically exceed this amount; a single depth perception apparatus (15 would be needed for one of our labs) from a well-known supplier of psychological equipment runs over $\$ 100$. Thus, this expenditure is not excessive, particularly because such equipment may be used for exercises in other laboratory classes (see Rowland \& Wesselhoft, 1998). We found that several student experimenters initially reported unusual BP recordings, probably due to improper positioning of the cuff and arm. This problem emphasizes the need for careful instruction and practice in the use of the monitors.

A final issue pertains to the sequencing of the stimuli, with relaxation first, followed by suspense. With the brief interstimulus interval used in this exercise, this order is critical to produce reliable changes in physiological measures across stimulus conditions. Specifically, the effects from the two types of stimulation (relaxation vs. suspense) are not symmetric. The suspense stimulus activates the sympathetic nervous system, and therefore its effects on physiology are more enduring than those of relaxation. As a result, reversing the order might obscure stimulus effects because physiological measures would fail to return to baseline before introduction of the relaxation stimulus. Such methodological issues regarding experimental control and design are worth raising as part of the discussion of the results and can add to the pedagogical value of the exercise. 


\section{References}

Greenwald, M. K., Cook, E. W., III, \& Lang, P. J. (1989). Affective judgment and psychophysiological response: Dimensional covariation in the evaluation of pictorial stimuli. Journal of Psychophysiology, 3, 51-64.

Hamilton, S. B., \& Knox, T. A. (1985). The colossal neuron: Acting out physiological psychology. Teaching of Psychology, 12, 153-156.

Harcum, E. R. (1988). Reaction time as a behavioral demonstration of neural mechanisms for a large introductory psychology class. Teaching of Psychology, 15, 208-209.

Jaffe, S., Lansing, S. (Producers), \& Lyne, A. (Director). (1987). Fatal attraction [Videotape]. (Available from Paramount Home Video)

Kemble, E. D. (1983). Insect predation by rodents: Some inexpensive experiments in comparative and physiological psychology. Teaching of Psychology, 10, 109-110.

Kirkpatrick, D. R. (1984). Age, gender, and patterns of common intense fears among adults. Behavior Research and Therapy, 22, $141-150$.

Lang, P. J., Levin, D. N., Miller, G. A., \& Kozak, M. J. (1983). Fear imagery and the psychophysiology of emotion. Journal of Abnormal Psychology, 92, 276-306.

Meditation [Videotape]. (1990). (Available from Francis/Nagle Release, 163 Amsterdam Avenue, Suite 207, New York, NY 10023)

Pierce, K. A., \& Kirkpatrick, D. R. (1992). Do men lie on fear surveys? Behavior Research and Therapy, 30, 415-418.

Rowland, D. L. (1995). The psychobiology of sexual arousal. In L. Diamant \& R. McAnulty (Eds.), The psychology of sexual orientation, behavior, andidentity (pp. 19-44). Westport, CT: Greenwood.
Rowland, D. L., \& Wesselhoft, T. (1998). The study of biobehavioral rhythms in a psychology laboratory course. Teaching of Psychology, 25, 106-109.

Solomon, P. R., Cooper, S., \& Pomerleau, D. (1988). Computer simulation of the neuronal action potential. Teaching of Psychology, $15,46-47$.

Webster, D. G., \& Muir, J. J. (1995). Presenting physiological measures: Effectiveness of demonstration versus lecture. Teaching of Psychology, 22, 192-195.

Wellman, P. J. (1984). Brown adipose tissue thermogenesis: A simple and inexpensive laboratory exercise in physiological psychology. Teaching of Psychology, 11, 115-116.

Wellman, P. J. (1985). On turning stereotaxic surgical failures into positive learning experiences in the undergraduate physiological psychology laboratory. Teaching of Psychology, 12, 228-229.

\section{Notes}

1. A packet of materials used for this laboratory exercise is available on request. We cannot provide videotape segments, but any comparable audiovisual relaxation tape (usually obtainable for $\$ 25$ or less) and any suspenseful 10 -min segment from a "thriller" videotape should suffice. For suspense in our demonstration, we used the sequence preceding and including the "stalking/bathroom" scene in Fatal Attraction (Jaffe, Lansing, \& Lyne, 1987).

2. Send correspondence to David L. Rowland, Department of Psychology, Valparaiso University, Valparaiso, IN 46383; e-mail: david.rowland@valpo.edu. 